\title{
A new definition of the Radar Cross Section suitable for surface wave propagation
}

\author{
Quentin HERBETTE*, Stéphane SAILLANT \\ Electromagnetism and Radar Department \\ ONERA, Université Paris Saclay \\ F-91123 Palaiseau, France \\ quentin.herbette@onera.fr
}

\begin{abstract}
This paper deals with a new definition of the Radar Cross Section suitable for surface wave propagation in the HF band. Indeed, it can be shown that the classical definition of the RCS cannot converge for this kind of propagation. This is an issue for the performance assessment of High Frequency Surface Wave Radars. Thanks to the analysis of different wave propagation models, the differences between the space wave propagation and surface wave propagation have been highlighted. The required modifications of the RCS should then be performed. The new definition is explained in the paper and it is shown that it is suitable for the surface wave propagation. Moreover, this new definition has been used for the computation of the RCS of 3D naval targets and the obtained results will be presented during the conference.
\end{abstract}

Keywords-HF Surface Wave Radar, Radar Cross Section

\section{INTRODUCTION}

For more than a decade, ONERA has been deploying surface wave radars. These radars operating in the HF band allow detections up to 270 miles. They represent a low cost solution for monitoring the Exclusive Economic Zone (EEZ), and they have been previously integrated in a European maritime surveillance system [1]. In order to forecast the performance of surface wave radars on their future deployment sites, there is an interest in developing a dedicated simulation software for surface wave radars. This code will require a database of RCS from different representative targets. However, the usual definition (1) of the RCS [2] is not suitable for surface wave propagation.

$$
\sigma=\lim _{R \rightarrow \infty} 4 \pi R^{2} \frac{\left|E_{S}\right|^{2}}{\left|E_{0}\right|^{2}}
$$

Indeed, with this kind of propagation the incident field $E_{0}$ on the target is not homogeneous or plane. In addition, the scattered field $E_{S}$ does not decay inversely with the distance $R$. In consequence a new definition of the RCS has been developed. Moreover, thanks to the electromagnetic simulation software FEKO it has been possible to demonstrate that this new definition is suitable for the surface wave propagation. In order to simulate realistic targets, various naval targets have been modeled using 3D modeling software.

Thanks to numerical simulations, in section II, it is shown that the usual RCS definition is not suitable for the surface wave propagation. In section III, the analysis of different propagation

\author{
Nicolas BOUREY, Muriel DARCES, \\ Florent JANGAL, Marc HÉLIER \\ Sorbonne Université, CNRS, Laboratoire de Génie Electrique et \\ Electronique de Paris, 75252, Paris, France \\ Université Paris-Saclay, CentraleSupélec, CNRS, Laboratoire \\ de Génie Electrique et Electronique de Paris, \\ 91192, Gif-sur-Yvette, France
}

models is explained. In section IV, the new definition of the RCS is introduced and is compared to the classical one.

\section{IRREVELANT RCS DEFINITION FOR SURFACE WAVE}

The first step is to show, by means of numerical simulation, that the classical definition of the RCS is not suitable for surface wave propagation. For that purpose, we are interested in the convergence of the RCS according to the distance. FEKO electromagnetic simulation software is applied here. It has the advantage of taking into account the surface wave via the use of Sommerfeld's integral formulas in the Green's functions. Solving the problem with the method of moments allows, in a limited time, to calculate the exact fields at a very long distance.

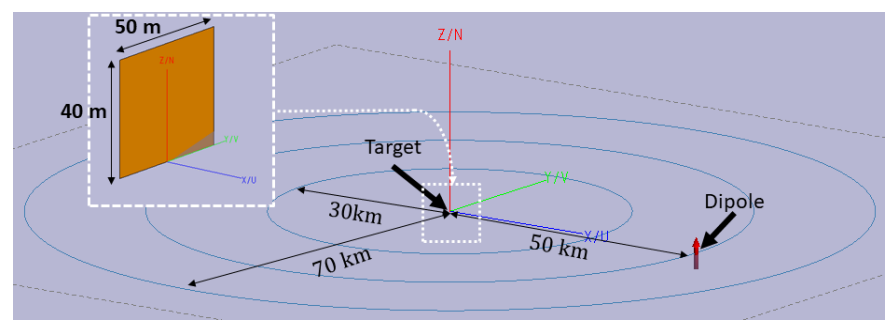

Fig. 1. Simulation scene of the scattering of a plate above the sea

The simulation scene can be seen in Fig.1: the target is a rectangular plate of $40 \mathrm{~m}$ high and $50 \mathrm{~m}$ wide. It has a zero thickness in the $x$ dimension. The lower medium is the sea $\left(\varepsilon_{r}=81, \sigma=5 \mathrm{~S} \cdot \mathrm{m}^{-1}\right)$, and the upper medium is the air.

The incident field on the plate is produced by a dipole, located at the interface, and radiating at $50 \mathrm{~km}$ from the target. Its radiation is identical to the one of the Bannister dipole [3].

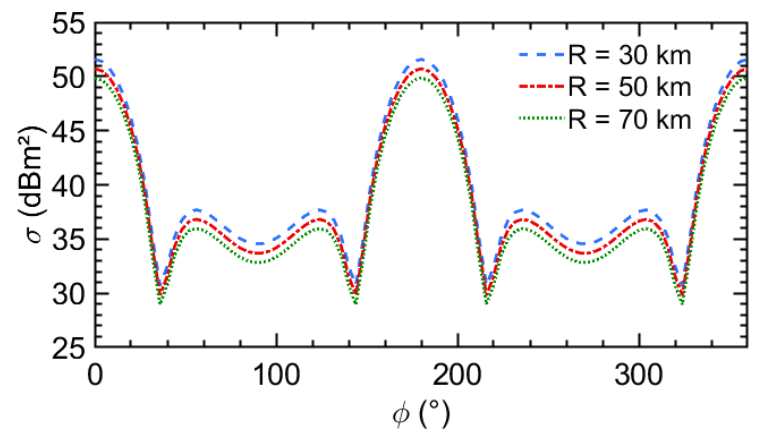

Fig. 2. Bistatic RCS $\sigma$ of a vertical plate in a surface wave propagation 
The scattered field is computed for all azimuthal angles at a distance $R$ of 30,50 and $70 \mathrm{~km}$ from the target at a height of $1 \mathrm{~m}$. The simulation frequency is $10 \mathrm{MHz}$. The classical formula of the RCS is applied to these data in order to compute the bistatic RCS of the target as shown in Fig.2.

It can be seen that the RCS does not converge according to the distance. This result was expected because the properties of space wave propagation and surface wave propagation are not the same.

\section{DIFFERENT PROPAGATION MODELS}

Despite a large number of propagation models described in the scientific literature, the issue of the convergence of the RCS for this specific propagation type does not seem to have been addressed yet. The analysis of different propagation models is necessary in order to define the main characteristics of surface wave propagation. The interest here is mainly focused on three propagations models. The first is the Bannister model, based on Norton's [4] work: it analytically separates the field in three components, direct wave, ground-reflected wave and the surface wave, radiated by a dipole placed at an arbitrary height above a plane half-space. The second is the Rotheram [5] model: this analytical model takes into account the roundness of the Earth and an exponential variation of the refractive index of the atmosphere. This model is the one recommended by the ITU. Finally, the last propagation model available is based on the parabolic wave equation: it allows in particular to take into account strong variations of the atmospheric refraction index.

The analysis of these three models made it possible to draw the three same conclusions on the structure of the surface wave, for the HF Band. First, the incident surface wave is not homogeneous or plane. Second, the scattered field does not decay inversely with the distance $R$, which makes the computation of the RCS divergent. Third, the distribution of the field, in amplitude and phase, is independent of the distance. This last conclusion is decisive for the new definition of a constant RCS suitable for the surface wave propagation. It shall be noticed that these conclusions are only valid at a long distance from the radiating source (i.e. over $30 \mathrm{~km}$ ) that is the typical case in HF surveillance.

\section{A NEW FORMULATION OF THE RCS FOR THE SURFACE WAVE PROPAGATION}

The proposed formulation of the new $\operatorname{RCS} \sigma_{S W}$ is provided by equation (2). It has a corrective term, the function $D$, which compensates for the surface wave amplitude variation. This dimensionless quantity makes it possible to take into account the specific evolution of the surface wave field according to the distance.

$$
\sigma_{S W}=\lim _{R \rightarrow \infty} 4 \pi R^{2} \frac{1}{D\left(\Gamma_{\|}, F(w)\right)} \frac{\left|E_{z_{s}}\right|^{2}}{\frac{1}{h_{\text {target }}} \int_{0}^{h_{\text {target }}}\left|E_{z_{i}}\right|^{2} d z}
$$

This function is mainly based on the work of Bannister, which has been adapted for the pursued goal. $E_{z_{s}}$ is the vertical scattered field, $E_{z_{i}}$ is the incident one and $h_{\text {target }}$ is the height of the target. This formulation is not destructive, which means that it does not change the angular variation of the RCS but it makes it possible to converge towards a unique value representative of the scattering potential of the target whatever the distance is. We then apply this formula to the data of the previous simulation. We observe in Fig.3 that the new RCS $\sigma_{S W}$ converges in distance, indeed the three curves are superimposed. This last result confirms that the new definition is well suited to this particular mode of surface wave propagation.

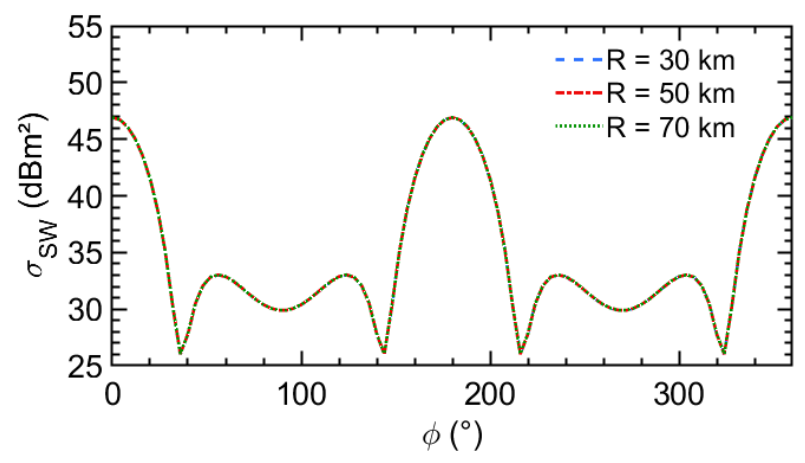

Fig. 3. Bistatic RCS $\sigma_{S W}$ of a vertical plate in a surface wave propagation

\section{CONCLUSION}

In this paper, it has been shown that the usual definition of the Radar Cross Section is not suitable for a surface wave propagation as it does not converge with regards to the distance. Thanks to the analysis of three analytical and numerical models, different conclusions on the surface wave propagation have been made. They enable us to determine a new definition of the RCS suitable for the surface wave propagation and independent of the observation distance. During the conference, some results on typical vessels will be presented. In the future, comparisons with measurements of previous and new prototype of surface wave radars developed by ONERA will be carried out.

\section{ACKNOWLEDGMENT}

DGA (French Ministry of Defense) is thanked for its contractual support to this work.

\section{REFERENCES}

[1] F. Jangal and M. Menelle, "French HFSWR contribution to the European integrated maritime surveillance system I2C," in IET International Radar Conference 2015, 2015, pp. 1-5, doi: 10.1049/cp.2015.1276.

[2] E. F. Knott, J. F. Shaeffer, and M. T. Tuley, Radar Cross Section. IET Digital Library, 2004.

[3] P. R. Bannister, "New Formulas That Extend Norton's Farfield Elementary Dipole Equations to the QuasiNearfield Range," 1984.

[4] K. A. Norton, "The Propagation of Radio Waves over the Surface of the Earth and in the Upper Atmosphere," Proc. Inst. Radio Eng., vol. 25, no. 9, pp. 1203-1236, Sep. 1937, doi: 10.1109/JRPROC.1937.228544.

[5] S. Rotheram, "Ground-wave propagation. Part 2: Theory for medium and long distances and reference propagation curves," IEE Proc. F - Commun. Radar Signal Process., vol. 128, no. 5, pp. 285-295, Oct. 1981, doi: 10.1049/ip-f1.1981.0047. 